\title{
Inertial sensors to quantify the pivot shift test in the treatment of anterior cruciate ligament injury
}

\author{
STEFANO ZAFFAGNINI ${ }^{1}$, NICOLA LOPOMO ${ }^{1,2}$, CECILIA SIGNORELLI $^{1,3}$ \\ GIULIO MARIA MARCHEGGIANI MUCCIOLI ${ }^{1}$, TOMMASO BONANZINGA ${ }^{1}$ \\ ALBERTO GRASSI ${ }^{1}$, FEDERICO RAGGI ${ }^{1}$, ANDREA VISANI ${ }^{1}$, MAURILIO MARCACCI $^{1}$ \\ ${ }^{1}$ Biomechanics and Technological Innovation Laboratory, Rizzoli Orthopaedic Institute, Bologna, Italy \\ 2 Laboratory of Nanobiotechnology (NaBi), Rizzoli Orthopaedic Institute, Bologna, Italy \\ ${ }^{3}$ Department of Electronics, Information and Bioengineering, Politecnico di Milano, Milan, Italy
}

\begin{abstract}
The main purpose of this article was to describe in detail, from the perspective of the clinical end user, a previously presented non-invasive methodology, applied in the treatment of anterior cruciate ligament injury, in which inertial sensors are used to quantify the pivot shift test. The outcomes obtained and relative considerations were compared with findings emerging from a review of the relevant updated literature. The detailed description here provided covers the system, the parameters identified and the testing procedure; it also includes the technical specifications of the hardware, the features introduced in the updated version of the software and the application of the system in clinical practice.

The comparison of the technical considerations and clinical results with the updated literature confirmed the system's optimal ergonomics, good reproducibility and clinical reliability.

The novel approach here analyzed has been shown to overcome the weaknesses of other available devices and systems. Therefore, since it can be considered a new paradigm in the quantification of pivot shift test, we can recommend its routine use in clinical practice.
\end{abstract}

\footnotetext{
Corresponding Author:

Nicola Lopomo, PhD

Biomechanics and Technological Innovation Laboratory,

Rizzoli Orthopedic Institute, Bologna, Italy

Via di Barbiano 1/10, 40136 Bologna, Italy

Tel: +390516366042

Fax: +39051583789

E-mail: n.lopomo@biomec.ior.it
}

Key words: acceleration, anterior cruciate ligament, knee kinematics, knee stability, pivot shift test, reconstruction.

\section{Introduction}

The anterior cruciate ligament (ACL) has been demonstrated to play a key role in providing knee stability and controlling joint kinematics (1).

Accordingly, ACL injury has been reported to be one of the most severe injuries in sports medicine (2), with an estimated incidence of approximately 30.000 injuries per year in Italy. This injury is seen across the young population but also in middle-aged patients and a high level of risk was observed in about $30 \%$ of Italian males and $20 \%$ of Italian females engaged in sports (3). ACL reconstruction has thus been found to be one of the most commonly performed procedures in sports medicine (4), having a critical impact on National Health Service (NHS) costs.

Short-term knee stability and functional outcome have usually been reported to be good-to-excellent in approximately 85 to $95 \%$ of patients. In the long term, however, ACL injury could be a cause of disability in about 30 to $50 \%$ of the young and productive population (4). Affected patients are indeed predisposed to subsequent injuries, muscle weakness and osteoarthritis (5). Therefore, ACL injury is a life-changing situation for many patients (6). Moreover, although correction of the joint laxity in the sagittal plane is usually the main objective of ACL reconstruction, the success of the surgery may well be compro- 
mised by residual rotational laxity which can affect the graft and may contribute to long-term joint degeneration (7-9). For these reasons a correct clinical assessment of knee joint laxity, with a view to predicting the functional behavior of the ACL, is a critical aspect of the management of the diagnosis-treatment-rehabilitation process.

The literature contains numerous clinical tests designed to objectively identify the ACL lesion, each with its own specificity and sensitivity towards the ligament tear (10-17). At present, the pivot shift test is the benchmark for ACL injury assessment, since it has been reported to be correlated with reduced sports activity and complete or partial tear of the ACL (18, 19). Historically, the pivot shift test was used for clinical evaluation of ACL deficiency and knee instability (18-20), and it is widely used for subjective assessment of rotatory knee laxity in combination with outcome scores such as the International Knee Documentation Committee score (21). However, although the pivot shift test has been widely investigated, a valid objective measurement is still lacking. The main problem is that the test itself involves combined loading of the joint, inducing movements in more than one degree of freedom during knee flexion-extension motion; this makes it difficult to determine a single synthetic parameter quantifying the pivot shift test outcome.

Recent reviews have highlighted the importance, in present-day clinical practice, of quantifying the pivot shift maneuver during the assessment of ACL injuries (22-24), also underlining the different technologies that have been developed to this end (25). In 2012, Lopomo et al. introduced and validated a novel methodology in which a three-dimensional acceleration signal, acquired by means of dedicated acceleration sensors and an integrated system, was used to quantify the pivot shift $(26,27)$.

The main purpose of the present report was to provide a description of this non-invasive methodology from the point of view of the clinical end user. In particular, the authors describe the practical use of this technology in a specific clinical condition (ACL injury) and also present literature data on its reliability and clinical usefulness. Support for the introduction of this system comes from the fact that the methodology seems to specifically introduce a new paradigm into the quantification of the pivot shift test.

\section{Equipment}

The system, an evolution of the one reported by Lopomo et al. $(26,27)$, consists of an inertial wireless sensor, linked to a tablet PC equipped with dedicated software (KiRA, Orthokey LTD, Lewes, Delaware, DE, USA) (Fig. 1).

The sensor embeds a three-axial accelerometer $( \pm 6 \mathrm{~g}$ range, with $g=9.80665 \mathrm{~m} / \mathrm{s}^{2}$ ) and three orthogonal gyroscopes ( $\pm 2000 \mathrm{dpm}$ range), for a total weight of $10 \mathrm{~g}$. The sampling rate is set at $110 \mathrm{~Hz}$ and the data transmission to the tablet $\mathrm{PC}$ is based on Bluetooth 2.0 standard. The tablet PC is a commercial model (Iconia Tab, W500 series, Acer, Taipei, Taiwan) that has Windows 7 (Microsoft Corporation, Redmond, Washington, USA) as its operating system. Dedicated software was developed by the company in order to manage both the communication with the sensor, the acceleration data acquisition and the patients' database.

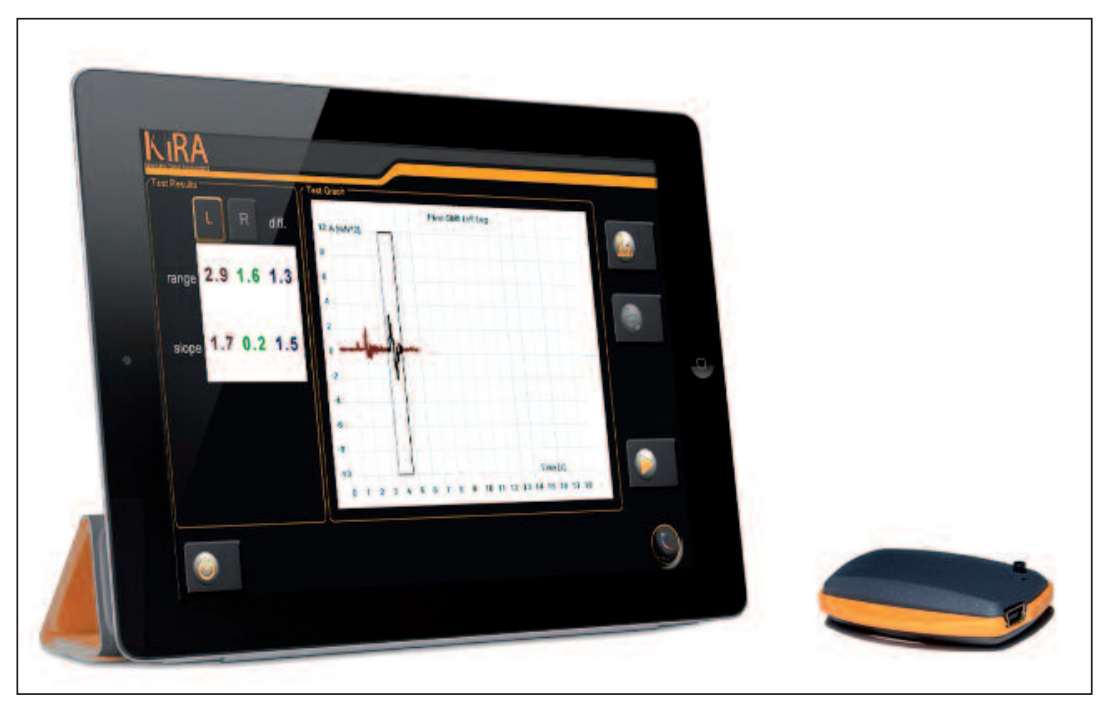

Fig. 1. Graphical representation of the described system (KiRA, Orthokey Ltd, Lewes, Delaware, DE, USA), showing the tablet PC (on the left) running the dedicated acquisition software, and the sensor (on the right). Courtesy of Orthokey Ltd, Lewes, Delaware, DE, USA. 


\section{Ergonomics}

Following the indications provided by Lopomo et al. (26), the sensor is mounted non-invasively on the patient's skin, close to the knee joint; it is securely fixed on the tibia, between the lateral aspect of the anterior tuberosity and Gerdy's tubercle, by means of a hypoallergenic band (Fig. 2). This position ensures optimal stability and minimizes skin artifacts during the maneuver. Moreover, this position is also justified by the fact that the lateral compartment of the knee joint has been found to be the one most influenced by the presence of the pivot shift phenomenon $(28,29)$.

\section{Software design and features}

The software running on the tablet PC, dedicated to communication with the sensor and to the acquisition of patient data, was developed by the manufacturer (Orthokey Ltd, Lewes, Delaware, DE, USA) in accordance with several specific requirements, both clinical and technical. Specifically, different features were incorporated into the software in order to facilitate the execution and the acquisition of the test. The tested version includes: automatic Bluetooth detection, built-in Bluetooth configuration, use of touchscreen technology to improve ergonomics, automatic extraction of pivot shift features, analysis of both the injured and the contralateral limb, a patient management tool, a camera-embedded system for reporting, and on-line report generation.

As regards the automatic extraction of the pivot shift features, as also reported by Signorelli et al. (30), the modulus of the spatial acceleration measured by the sensor is used as the base signal against which to identify the different pivot shift events. Specifically, recognition of the pivot shift events is based on calculation of the sample-by-sample Pearson's correlation coefficient obtained between a specific template - defined on the set of trial patients - and the corresponding portion of the signal (30). Once the pivot shift events have been defined, the system is able to extract the components of the acceleration reached, specifically identifying the range of obtained acceleration (i.e. the amount of acceleration between maximum and minimum values within the selection window) and the mean slope of the corresponding curve, which specifically provides an indication of the smoothness of the identified phenomenon (Fig. 3).

If the automatic identification of the pivot shift event is not good or if the end user realizes that the identified event is not associated with the best execution of the maneuver, the end user himself can move the identification window on the screen and manually highlight the correct portion of the signal, in order to obtain the correct corresponding numerical values.

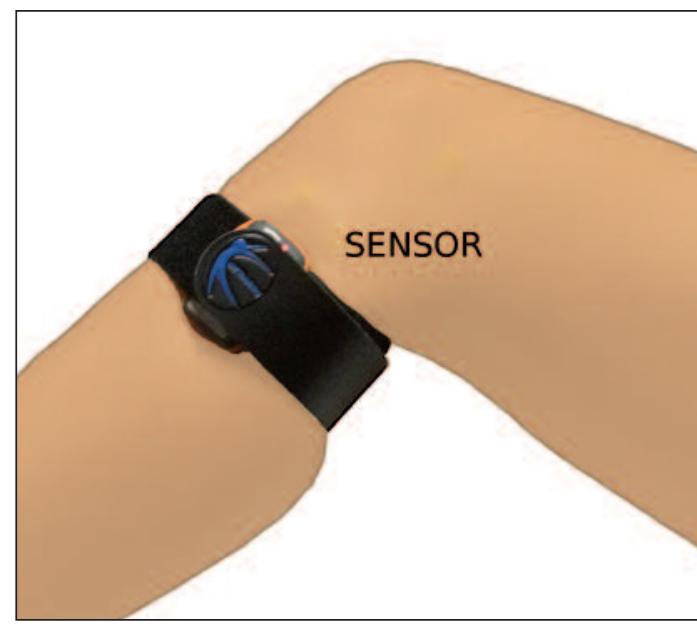

Fig. 2. Acceleration sensor set-up: the sensor was positioned between the lateral aspect of the anterior tuberosity and Gerdy's tubercle.

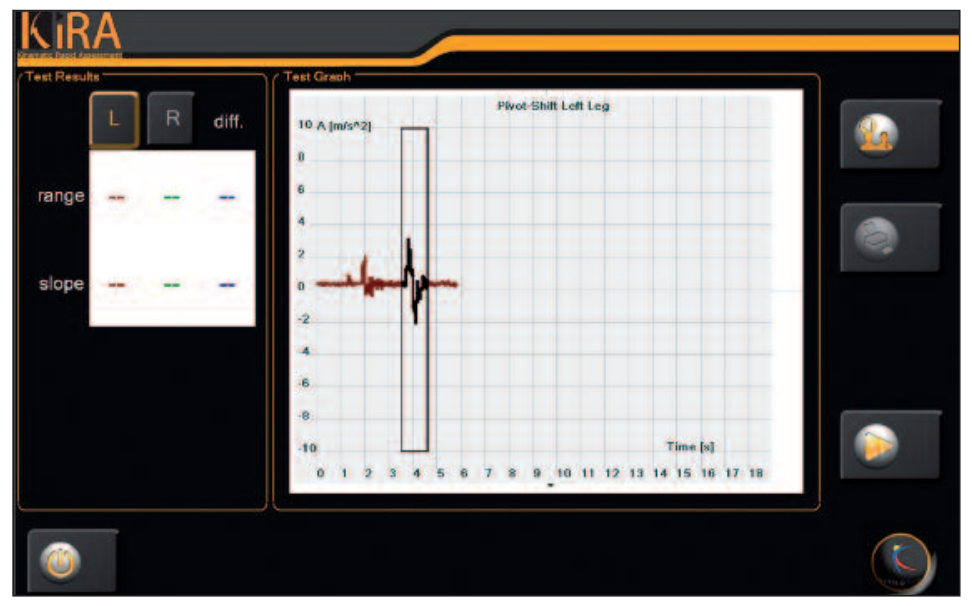

Fig. 3. Typical acceleration trend during a pivot shift test acquisition The parameters range and slope are reported on the right side of the acquisition panel. The comparison between the injured and the contralateral limb can be performed immediately. 


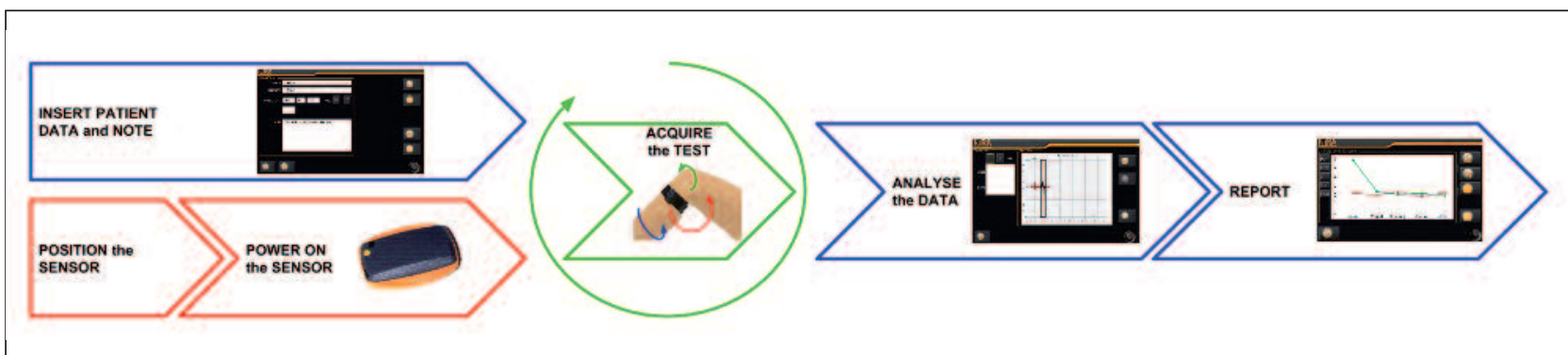

Fig. 4. Workflow for pivot shift acquisition and data management.

\section{Testing procedure}

Once the sensor has been fixed, the patient is assessed in the manner described in the literature $(18,19,31)$ and using the standardized maneuver as reported by Hoshino et al. (32). In particular the patient lies on the couch in supine position with both limbs extended and the tester, who remains laterally positioned with respect to the patient, performs the following steps: i) grasps the patient's limb by the ankle, lifting it; ii) grasps the foot and rotates the tibia internally (about 20 degrees); iii) grasps, with the other hand, the lateral portion of the leg at the level of the superior tibiofibular joint and increases the force applied to obtain internal rotation; iv) while keeping the tibia internally rotated, applies a valgus stress on the knee and slowly flexes the joint; v) having reached 60-70 degrees of flexion, stops the movement; vi) returns the limb to the initial neutral position. Standardization of the procedure is fundamental to reduce both intra- and inter-tester variability (Fig. 4). Furthermore, using this system, it is possible to track the evolution of the laxity of each specific patient (Fig. 5).

\section{Literature review}

Lopomo et al. (26), analyzing 66 patients, found the method to show good reliability in identifying the pivot shift phenomenon when used on the injured limb (interclass correlation coefficient above $90 \%$ for the acceleration range and above $80 \%$ for the slope). Moreover, they reported that, compared with the healthy limbs, the injured knees showed a higher acceleration range (with a statistical difference of $1.6 \pm$ $1.5 \mathrm{~m} / \mathrm{s}^{2}$ ) and a higher slope (with a statistical difference of $8.6 \pm 13.7 \mathrm{~m} / \mathrm{s}^{3}$ ).

The same research group also analyzed the reliability of the method, comparing it with a navigation system (27). In particular they evaluated the position of the sensor in 15 consecutive surgeries, reporting a good value of average root mean square (RMS) error in testretest positioning (less than $6 \mathrm{~mm}$ ). Moreover, mean RMS displacement due to soft tissue artifacts was reported to be less than $5 \mathrm{~mm}$. They also reported a mean acceleration waveform correlation of about 0.9 in inter-patient similarity analysis and a good positive correlation between the measurements performed by the inertial sensor and the antero-posterior acceleration value estimated by the navigation system.

Recent studies have also analyzed, on an in vitro setup, the reliability of use of the inertial sensor in discrimi-

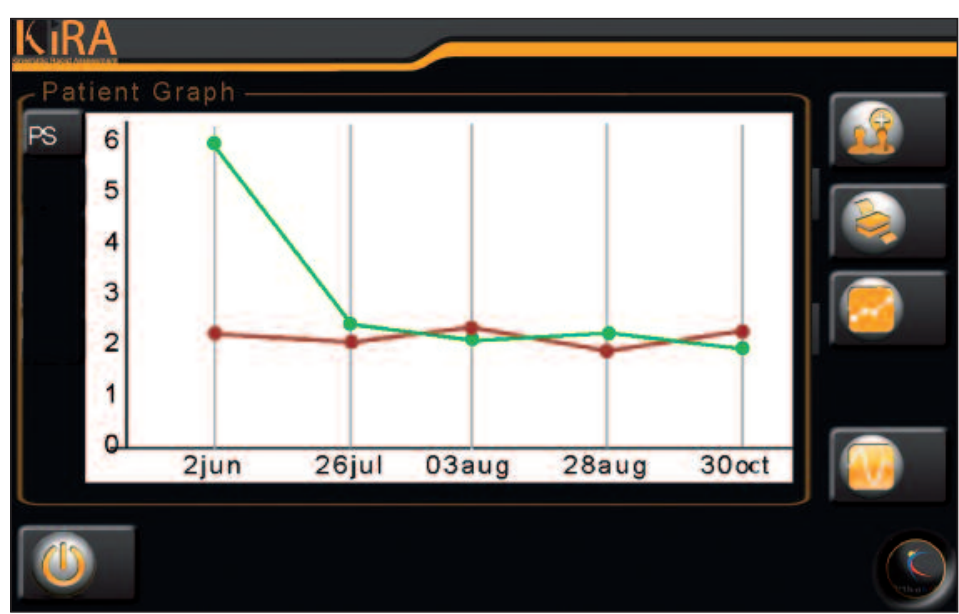

Fig. 5. Example of pivot shift quantitative analysis tracking during follow-ups. 
nating the grade of the pivot shift, compared with an electromagnetic system used as a gold standard (33, 34). Ahldén et al. (33), reported a good correlation between the slope and the maximum acceleration measured by the sensor and the average pivot shift grade. Moreover, Araujo et al. (34) found that the inertial sensor demonstrated from moderate to good correlation with the reference measurement for the acceleration parameter.

From the more clinical point of view, Berruto et al. (35), in a case series of 100 patients analyzed by three physicians with different levels of expertise, reported that the use of the presented methodology was both promising and reliable. They also highlighted that the analyzed efficacy was strictly related to a learning curve and to the proper execution of the pivot shift test. In any case, the proposed methodology has the inherent value of being easy to set up and easy to use in both the clinic and the operating room.

\section{Conclusions}

Quantification of the pivot shift phenomenon can be considered one of the major issues facing the orthopedists involved in ACL surgery.

A previously proposed non-invasive, portable and user-friendly methodology $(26,27)$ was here analyzed from the point of view of the clinical end user, in order to support the usefulness of this novel approach in the quantification of the pivot shift test. Details about the technology, the practical procedure and the method used for the data analysis were presented. In particular, the specifically designed and developed features embedded in the system used have been found to be effective in clinical practice. The system's ergonomics, here highlighted, ensured ease of use for both acquisition and patient data management. Moreover, literature reports have also highlighted the ability of the setup to minimize soft tissue artifacts, which are not a negligible problem when considering the routine use of this device. Finally, from the clinical point of view, the defined methodology allowed the definition of a brief set of parameters able to discriminate pathological behavior in ACL-injured knees. The literature also contained good results concerning the reliability of the system for use in clinical practice and applications. Summarizing, from the point of view of the clinical end user, this novel approach has been shown to overcome the weaknesses of other devices, including navigation systems $(29,36,37)$ and electromagnetic trackers (38-41), and is thus suitable for routine use in clinical practice, since it seems to constitute a new paradigm in the quantification of the pivot shift test.

\section{Acknowledgments}

All the authors would like to acknowledge the anesthetists and all the staff of the "Clinica Ortopedica e Traumatologica II”, Rizzoli Orthopedic Institute, for their assistance during this study. NL and AV would very much like to thank Mr. Emil Ferretti for his support in managing the Laboratory of Nanobiotechnology and guaranteeing its "technological supremacy".

\section{References}

1. Fukubayashi T, Torzilli PA, Sherman MF, et al. An in vitro biomechanical evaluation of anterior-posterior motion of the knee. Tibial displacement, rotation, and torque. J Bone Joint Surg Am. 1982;64:258-264.

2. Möller E, Weidenhielm L, Werner S. Outcome and knee-related quality of life after anterior cruciate ligament reconstruction: a long-term follow-up. Knee Surg Sports Traumatol Arthrosc. 2009; 17:786-794.

3. Calvisi V, De Vincentiis B, Palumbo P, et al. Health-related quality of life in patients with anterior cruciate ligament insufficiency undergoing arthroscopic reconstruction: a practice-based Italian normative group in comorbid-free patients. J Orthop Traumatol. 2008;9:233-238.

4. Ferretti A, Monaco E, Giannetti S, et al. A medium to longterm follow-up of ACL reconstruction using double gracilis and semitendinosus grafts. Knee Surg Sports Traumatol Arthrosc. 2011;19:473-478.

5. Beynnon BD, Uh BS, Johnson RJ, et al. Rehabilitation after anterior cruciate ligament reconstruction: a prospective, randomized, double-blind comparison of programs administered over 2 different time intervals. Am J Sports Med. 2005; 33:347-359.

6. Månsson O, Kartus J, Sernert N. Health-related quality of life after anterior cruciate ligament reconstruction. Knee Surg Sports Traumatol Arthrosc. 2011;19:479-487.

7. Beard DJ, Dodd CA, Trundle HR, et al. Proprioception enhancement for anterior cruciate ligament deficiency. A prospective randomised trial of two physiotherapy regimes. J Bone Joint Surg Br. 1994;76:654-649.

8. Georgoulis AD, Papadonikolakis A, Papageorgiou CD, et al. Three-dimensional tibiofemoral kinematics of the anterior cruciate ligament-deficient and reconstructed knee during walking. Am J Sports Med. 2003;31:75-79. 
9. Tashman S, Collon D, Anderson K, et al. Abnormal rotational knee motion during running after anterior cruciate ligament reconstruction. Am J Sports Med. 2004;32:975-983.

10. Jensen K. Manual laxity tests for anterior cruciate ligament injuries. J Orthop Sports Phys Ther. 1990;11:474-481.

11. Scholten RJ, Opstelten W, van der Plas CG, et al. Accuracy of physical diagnostic tests for assessing ruptures of the anterior cruciate ligament: a meta-analysis. J Fam Pract. 2003;52: 689-694.

12. Ostrowski J. Accuracy of 3 diagnostic tests for anterior cruciate ligament tears. J Athl Train. 2006;41:120-121.

13. Graham GP, Johnson S, Dent CM, et al. Comparison of clinical tests and the KT1000 in the diagnosis of anterior cruciate ligament rupture. Br J Sports Med;1991;25:96-97.

14. Katz JW, Fingeroth RJ. The diagnostic accuracy of ruptures of the anterior cruciate ligament comparing the Lachman test, the anterior drawer sign, and the pivot shift test in acute and chronic knee injuries. Am J Sports Med. 1986;14:88-91.

15. Tibone JE, Antich TJ, Fanton GS, et al. Functional analysis of anterior cruciate ligament instability. Am J Sports Med. 1986; 14:276-284.

16. Benjaminse A, Gokeler A, van der Schans CP. Clinical diagnosis of an anterior cruciate ligament rupture: a meta-analysis. J Orthop Sports Phys Ther. 2006;36:267-288.

17. Johnson RJ. The anterior cruciate ligament problem. Clin Orthop Relat Res. 1983;(172):14-18.

18. Galway HR, MacIntosh DL. The lateral pivot shift: a symptom and sign of anterior cruciate ligament insufficiency. Clin Orthop Relat Res. 1980;(147):45-50.

19. Jakob RP, Stäubli HU, Deland JT. Grading the pivot shift. Objective tests with implications for treatment. J Bone Joint Surg Br. 1987;69:294-299.

20. Jakob RP, Hassler H, Staeubli HU. Observations on rotatory instability of the lateral compartment of the knee. Experimental studies on the functional anatomy and the pathomechanism of the true and the reversed pivot shift sign. Acta Orthop Scand Suppl. 1981;191:1-32.

21. Hefti F, Müller W, Jakob RP, et al. Evaluation of knee ligament injuries with the IKDC form. Knee Surg Sports Traumatol Arthrosc. 1993;1:26-234.

22. Lane CG, Warren R, Pearle AD. The pivot shift. J Am Acad Orthop Surg. 2008;16: 679-688.

23. Ahldén M, Hoshino Y, Samuelsson K, et al. Dynamic knee laxity measurement devices. Knee Surg Sports Traumatol Arthrosc. 2012;20: 621-632.

24. Zaffagnini S, Marcheggiani Muccioli GM, Lopomo N, et al. Can the pivot-shift be eliminated by anatomic double-bundle anterior cruciate ligament reconstruction? Knee Surg Sports Traumatol Arthrosc. 2012;20:743-751.

25. Lopomo N, Zaffagnini S, Amis AA. Quantifying the pivot shift test: a systematic review. Knee Surg Sports Traumatol Arthrosc. 2013;21:767-783.

26. Lopomo N, Zaffagnini S, Signorelli C, et al. An original clinical methodology for non-invasive assessment of pivot-shift test. Comput Methods Biomech Biomed Engin. 2012;15: 1323-1328.
27. Lopomo N, Signorelli C, Bonanzinga T, et al. Quantitative assessment of pivot-shift using inertial sensors. Knee Surg Sports Traumatol Arthrosc. 2012;20:713-717.

28. Bedi A, Musahl V, Lane C, et al. Lateral compartment translation predicts the grade of pivot shift: a cadaveric and clinical analysis. Knee Surg Sports Traumatol Arthrosc. 2010; 18:1269-1276.

29. Lopomo N, Zaffagnini S, Bignozzi S, et al. Pivot-shift test: analysis and quantification of knee laxity parameters using a navigation system. J Orthop Res. 2010;28:164-169.

30. Signorelli C, Lopomo N, Bignozzi S, et al. Automatic screening of acceleration signal during pivot-shift test based on Pearson's correlation coefficient. Proc. BIOSTEC, BIOSIGNALS 2010. Int. Conf. Bio-inspired Syst. Signal Process. Abstr.

31. Slocum DB, James SL, Larson RL, et al. Clinical test for anterolateral rotary instability of the knee. Clin Orthop Relat Res. 1976; (118):63-69.

32. Hoshino Y, Araujo P, Ahlden M, et al. Standardized pivot shift test improves measurement accuracy. Knee Surg Sports Traumatol Arthrosc. 2012;20:732-736.

33. Ahldén M, Araujo P, Hoshino Y, et al. Clinical grading of the pivot shift test correlates best with tibial acceleration. Knee Surg Sports Traumatol Arthrosc. 2012;20:708-712.

34. Araujo PH, Ahlden M, Hoshino Y, et al. Comparison of three non-invasive quantitative measurement systems for the pivot shift test. Knee Surg Sports Traumatol Arthrosc. 2012; 20:692-697.

35. Berruto M, Uboldi F, Gala L, et al. Is triaxial accelerometer reliable in the evaluation and grading of knee pivot-shift phenomenon? Knee Surg Sports Traumatol Arthrosc. 2013;21: 981-985.

36. Bignozzi S, Zaffagnini S, Lopomo N, et al. Clinical relevance of static and dynamic tests after anatomical double-bundle ACL reconstruction. Knee Surg Sports Traumatol Arthrosc. 2010;18:37-42.

37. Lopomo N, Bignozzi S, Zaffagnini S, et al. Quantitative correlation between IKDC score, static laxity, and pivot-shift test: a kinematic analysis of knee stability in anatomic doublebundle anterior cruciate ligament reconstruction. Oper Tech Orthop. 2008;18:185-189.

38. Kuroda R, Hoshino Y, Nagamune K, et al. Intraoperative measurement of pivot shift by electromagnetic sensors. Oper Tech Orthop. 2008;18:190-195.

39. Amis AA, Cuomo P, Rama RBS, et al. Measurement of knee laxity and pivot-shift kinematics with magnetic sensors. Oper Tech Orthop. 2008;18:196-203.

40. Labbe DR, Deguise J, Godbout V, et al. Development of an objective measurement tool for the pivot shift phenomenon of the knee. J Biomech. 2008;41:S207-S207.

41. Labbe DR, de Guise JA, Godbout V, et al. Accounting for velocity of the pivot shift test manoeuvre decreases kinematic variability. Knee. 2011;18:88-93. 